“C 2018 IEEE. Personal use of this material is permitted. Permission from IEEE must be obtained for all other uses, in any current or future media, including reprinting/republishing this material for advertising or promotional purposes, creating new collective works, for resale or redistribution to servers or lists, or reuse of any copyrighted component of this work in other works." 


\title{
Optimal Sparsely Distributed Static Output Feedback For Publisher/Subscriber Networked Systems With Parametric Uncertainties
}

\author{
Ahmadreza Argha ${ }^{\dagger}$, Steven W. Su${ }^{\star}$ and Branko Celler ${ }^{\dagger}$
}

\begin{abstract}
This paper develops a framework for the design of $\mathscr{H}_{2}$-based block row/column-sparse distributed static output feedback controllers for interconnected systems with polytopic uncertainties. The application of the proposed method will be in the networked systems using publisher/subscriber communication scheme in order to optimally select a subset of available publishers and/or subscribers in the network. We propose to incorporate two additional terms, that penalise the number of publishers and subscribers, into the optimisation index function, and then employ an explicit scheme and an iterative process to deal with this problem. A numerical example is used to demonstrate the effectiveness of the proposed approach in the simultaneous identification of favourable networks topologies and design of controller strategy.
\end{abstract}

Index Terms- $\mathscr{H}_{2}$ block row/column-sparse static output feedback (SOF) problem; publisher/subscriber networked systems; polytope uncertain systems, linear matrix inequality.

\section{INTRODUCTION}

The modern practical systems such as power distribution networks and transportation systems can be considered as large-scale interconnected dynamical systems, for which decentralised and distributed control schemes have been proposed. The main idea behind the decentralised control scheme is to use only the local state information in order to control the subsystems and thus there is no control network. This can be effective only when the interconnections between the subsystems are not strong [1], [2]. When the interconnections are strong, utilising distributed control frameworks has been considered. In this strategy, each subsystem can exploit local states as well as some of the other subsystems states. Hence, compared to the decentralised control scheme, distributed control scheme can ensure the stability of large scale interconnected systems in the presence of stronger subsystem interconnections [3]. Meantime, it also has less complexity and improved computational aspects compared to the centralised control scheme.

In interconnected systems, the structure of the distributed controller network is usually restricted due to a number of factors such as implementation-related concerns and communication costs. This issue in distributed systems is also referred to as information pattern, which means that, unlike traditional distributed control schemes in which all

\footnotetext{
$\doteqdot$ A. Argha and B. Celler are with the School of Electrical Engineering and Telecommunications, University of New South Wales, Sydney, NSW 2052 Australia.

$\{a \cdot$ argha and b.celler\}@unsw.edu.au

${ }^{\star}$ S. W. Su are with Faculty of Engineering and Information Technology, University of Technology, Sydney, PO Box 123, Broadway, NSW 2007, Australia.

steven.su@uts.edu.au
}

the involving sub-controllers share the same information, the sub-controllers can share or receive different information [4]. Since the fully distributed controller structure is not always feasible, one may consider the design of distributed control systems with imposing a priori constraints on communication network structure. Alternatively, another choice for the distributed control systems is to design a control network with the minimum number of communication links while satisfying a global control objective [5], [6]. Indeed, a trade off between the control performance and sparsity of the feedback gain matrices should be considered [7]-[9].

Basically, to address network sparsification problem, one has to, in worst case, check all possible topologies, implying an exhaustive search for a number of configurations that can grow exponentially with the number of communication links. This is practically intractable and impossible to perform. As explained in [10], to avoid performing an exhaustive search, a trade-off can be made either in the choice of the search strategy or in the choice of the selection criterion. Another alternative to avoid solving a combinatorial problem is to consider a multi-objective problem of controller structure and control law co-design by incorporating secondary cost functions which are convex approximations of the original $\ell_{0}$-quasi-norms and can promote sparsity of the distributed controller, into a main cost function, which represents a performance specification of the closed-loop system [7], [11]. The reweighted $\ell_{1}$ (REL1) norm algorithms can further be employed to identify the sparse optimal feedback gain [7]. The weights (entries of the weighting matrix), in the REL1 algorithms are updated at each step inversely proportional to the strength of individual entries of feedback gain in the previous step. This method has successively been applied to the applications that the sparsity is required to be achieved at the entry-wise level, i.e. minimising the number of communication links in distributed control networks exploiting the socalled bilateral communication scheme [4]. However, the current REL1 algorithms have shortcomings for the cases that the sparsity is noted at a group (e.g. block row or column) level, where the strength of groups of variables (block entries of feedback gain) should be considered. Specifically, block row/column sparse feedback gains have an application in the so-called diffusion based networks [4], in which a published information in the communication network is available to the sub-controllers that are subscriber. In this case, the objective is to minimise the number of subscribers/publishers in the system rather than the number of bilateral communication links. This is obviously equivalent to exploring for feedback gains with maximum number of block rows/columns with 
zero off-diagonal blocks. In this manuscript, we propose a multi-objective optimisation problem by incorporating two secondary index functions which penalise the number of block rows/columns with non-zero off-diagonal blocks into the main cost function. Then, in order to deal with the underlying problem, we develop an iterative process, using the relaxed block row/column sparsity promoting penalty functions to penalise the number of publishers and subscribers employed in the control network simultaneously.

The controller strategy proposed in this paper is static output feedback (SOF) which means, unlike most of the work in the current literature, it does not require all the system states to be available, and instead it only uses the available sensor outputs. Additionally, the overall networked system considered in this work is assumed to involve parametric uncertainties. Hence, this paper develops a novel framework for the design of $\mathscr{H}_{2}$-based sparse block row/column-wise SOF for polytope uncertain systems. An advantage of the proposed SOF for polytope uncertain systems is that it is applicable to the networked systems whose output matrix involves parametric uncertainty; cf. [12].

Notation: We denote a (block) matrix with (block) entries $\Phi_{i j}, \quad i=1, \cdots, r, j=1, \cdots, r$, as $\left[\Phi_{i j}\right]_{r \times r}$. Furthermore, $\operatorname{diag}\left[\Phi_{i}\right]_{i=1}^{r}$ is used to denote a (block) diagonal matrix with (block) entries $\Phi_{i}, i=1, \cdots, r$. Moreover, $\operatorname{col}\left(v_{i}(t)\right)_{i=1}^{r}$ represents a (block) vector with (block) entries $v_{i}(t), i=$ $1, \cdots, r$.

\section{Problem Statement And Preliminaries}

\section{A. Problem statement}

Consider the following large-scale linear-time-invariant (LTI) networked system consisting of $h$ subsystems:

$$
\left\{\begin{array}{l}
\dot{x}_{i}(t)=A_{i} x_{i}(t)+\sum_{j=1, j \neq i}^{h} A_{i j} x_{j}(t)+B_{2, i} u_{i}(t)+B_{1, i} w_{i}\left(x_{i}\right), \\
z_{i}(t)=C_{z, i} x_{i}(t)+\sum_{j=1, j \neq i}^{h} C_{z, i j} x_{j}(t)+D_{z, i} u_{i}(t), \\
y_{i}(t)=C_{i} x_{i}(t),
\end{array}\right.
$$

where $x_{i} \in \mathbb{R}^{n_{i}}, y_{i} \in \mathbb{R}^{p_{i}}, z_{i} \in \mathbb{R}^{q_{i}}$ and $u_{i} \in \mathbb{R}^{m_{i}}$ are the system state vector, output vector, performance output vector, and control input vector of the $i$-th subsystem, respectively. The matrices in (1) are constant and of appropriate dimensions. Here, $A_{i j}, i, j=1, \cdots, h, j \neq i$ denotes the interactions between the subsystems $\left(A_{i}, i=1, \cdots, h\right)$, i.e., $A_{i j}=0$ if the sub-system $j$ does not influence directly the sub-system $i$. Without loss of generality, it is also assumed that $m_{i} \leq$ $q_{i} \leq n_{i}$, and $\operatorname{rank}\left(B_{2, i}\right)=m_{i} . w_{i}(t) \in \mathbb{R}^{m_{w_{i}}}$ is the external disturbance of the system. Define

$$
\begin{aligned}
& x(t):=\operatorname{col}\left(x_{i}(t)\right)_{i=1}^{h} \in \mathbb{R}^{n}, u(t):=\operatorname{col}\left(u_{i}(t)\right)_{i=1}^{h} \in \mathbb{R}^{m}, \\
& y(t):=\operatorname{col}\left(y_{i}(t)\right)_{i=1}^{h} \in \mathbb{R}^{p}, w(x):=\operatorname{col}\left(w_{i}(t)\right)_{i=1}^{h} \in \mathbb{R}^{m_{w}}, \\
& z(t):=\operatorname{col}\left(z_{i}(t)\right)_{i=1}^{h} \in \mathbb{R}^{q},
\end{aligned}
$$

and

$$
\begin{aligned}
A & :=\operatorname{diag}\left[A_{i}\right]_{i=1}^{h}+\left[A_{i j}\right]_{h \times h}, B_{2}:=\operatorname{diag}\left[B_{2, i}\right]_{i=1}^{h}, \\
B_{1} & :=\operatorname{diag}\left[B_{1, i}\right]_{i=1}^{h}, C:=\operatorname{diag}\left[C_{i}\right]_{i=1}^{h}, \\
C_{z} & :=\operatorname{diag}\left[C_{z, i}\right]_{i=1}^{h}+\left[C_{z, i j}\right]_{h \times h}, D_{z}:=\operatorname{diag}\left[D_{z, i}\right]_{i=1}^{h},
\end{aligned}
$$

in which $A_{i i}=0$ and $C_{z, i i}=0$. Using (1), (2) and (3), the overall network can be presented as the following state space realisation:

$$
\left\{\begin{array}{l}
\dot{x}(t)=A x(t)+B_{2} u(t)+B_{1} w(x) \\
z(t)=C_{z} x(t)+D_{z} u(t) \\
y(t)=C x(t)
\end{array}\right.
$$

Let the overall system matrices in (4) lie within the polytope

$$
\begin{aligned}
\Delta \triangleq & \left\{\left(A(\lambda), B_{2}(\lambda), B_{1}(\lambda), C(\lambda)\right) \mid\left(A, B_{2}, B_{1}, C\right)\right. \\
& \left.=\sum_{l=1}^{\mathscr{N}} \lambda_{l}\left(A_{l}, B_{2, l}, B_{1, l}, C_{l}\right), \quad \lambda_{l} \geq 0, \sum_{l=1}^{\mathscr{N}} \lambda_{l}=1\right\},
\end{aligned}
$$

where $\mathscr{N}$ denotes the number of vertices. It is assumed that $\left(A_{l}, B_{2, l}\right)$ are stabilisable and there exists a static gain $K_{y}$ such that $A_{l}+B_{2, l} K_{y} C_{l}$ are Hurwitz.

Most of the frameworks developed in the literature of distributed control design for networked systems are applicable to the systems whose communication network topology is built based on the so-called bilateral communication scheme, in which the sub-systems have bilateral communications. Hence, for example, seeking for a sparse feedback gain is equal to utilising less communication links in the control network. Nevertheless, these frameworks have nothing to do with the so-called publisher/subscriber communication scheme; see Fig. 1. The diffusion based networks (see e.g. [4]), such as the factory instrumentation protocol (see e.g. EN 50170 and IEC 61158/IEC 61784 standards), is significantly different from the bilateral one. In this communication scheme, a published information in the communication network is available to the sub-controllers that are subscriber. In this paper, our major objective is the minimisation of the number of subscribers and/or publishers in the system. This is equivalent to exploring for feedback gains with maximum number of block rows/columns with zero offdiagonal blocks. More specifically, the main objective of this paper can be stressed as the following problem:

Problem 1: Given a networked system with the state space representation in Equation (4), select a subset of available publishers/subscribers and simultaneously find a distributed controller that employs only the available sparse information while minimising the degradation of an optimisation metric, say $\mathscr{H}_{2}$ norm of the overall closed-loop transfer function from $w$ to $z$, relative to the case where all the system information are exploited.

To address Problem 1, we firstly construct a framework for the design of a controller which uses a priori specified subset of system information. This framework can be employed to cope with different control network topologies. 


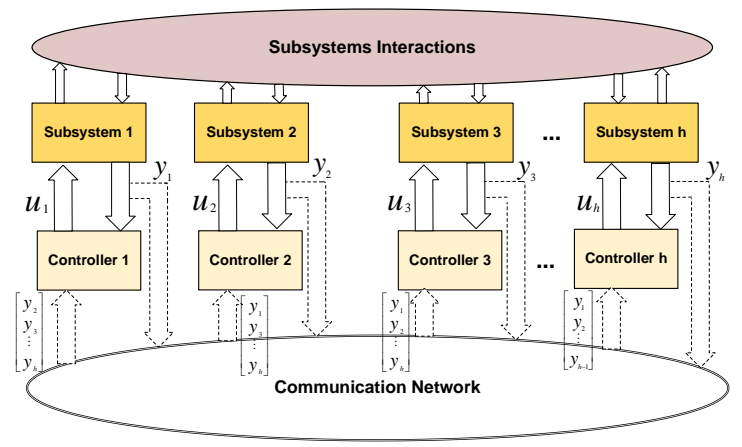

Fig. 1. Networked System Architecture

Problem 2: Given a networked system with the state space representation in Equation (4), find a distributed controller employing a priori specified subset of system information which minimises an optimisation metric, say $\mathscr{H}_{2}$ norm of the overall closed-loop transfer function from $w$ to $z$.

As the system in (1) or (4) involves the parametric uncertainty, we need to develop a framework for the design of sparsely distributed controllers for networked systems employing publisher/subscriber communication scheme such that it can deal with the existing parametric uncertainties. For this purpose, the problem of structured static output feedback synthesis with a $\mathscr{H}_{2}$ performance specification is primarily considered. Before doing so, we present some useful definitions.

Definition 1: A matrix whose elements are either 0 or 1 is said to be a structure matrix. Let $\Lambda=\left[\Lambda_{i j}\right]_{m \times n}$ be a block matrix with $\Lambda_{i j} \in \mathbb{R}^{a_{i} \times b_{j}}$, then the structure matrix of $\Lambda$ is obtained as $\mathrm{S}(\Lambda) \triangleq\left[\gamma_{i j}\right]_{m \times n}$ with

$$
\gamma_{i j}= \begin{cases}0 & \text { if } \Lambda_{i j}=0 \\ 1 & \text { otherwise. }\end{cases}
$$

Definition 2: Two matrices $\Lambda_{1}$ and $\Lambda_{2}$ are structurally the same if $S\left(\Lambda_{1}\right)=S\left(\Lambda_{2}\right)$.

Definition 3: The matrix $\Lambda_{1}$ with $\mathrm{S}\left(\Lambda_{1}\right) \triangleq\left[\gamma_{i j}^{1}\right]_{m \times n}$ is structurally subset of $\Lambda_{2}$ with $\mathrm{S}\left(\Lambda_{2}\right) \triangleq\left[\gamma_{i j}^{2}\right]_{m \times n}$ while $\gamma_{i j}^{2}-$ $\gamma_{i j}^{1} \geq 0$. We denote this as $\mathrm{S}\left(\Lambda_{1}\right) \subseteq \mathrm{S}\left(\Lambda_{2}\right)$.

Definition 4: A block matrix $\tilde{\Lambda}$ is said to be sparse block row-wise (column-wise) if its structure matrix $\tilde{\Gamma}$, i.e. $\mathrm{S}(\tilde{\Lambda})=$ $\tilde{\Gamma}$, includes (at least) one row (column) of all zeros.

\section{B. LMI-Based $\mathscr{H}_{2}$ State Feedback Design for Polytope Un- certain Systems}

Lemma 1: The following three statements, involving $X>$ $0, Z>0$, a general matrix variable $V$ are equivalent.

i) $\exists \quad K$ such that $A+B_{2} K$ is stable and $\left\|\left(C_{z}+D_{z} K\right)\left(s I-A-B_{2} K\right)^{-1} B_{1}\right\|_{2}^{2}<\gamma$. ii) $\exists X>0$ and $Z>0$ such that

$$
\begin{aligned}
& {\left[\begin{array}{cc}
A X+B_{2} Y+X A^{T}+Y^{T} B_{2}^{T} & \star \\
C_{z} X+D_{z} Y & -\gamma I
\end{array}\right]<0,} \\
& {\left[\begin{array}{cc}
-Z & \star \\
B_{1} & -X
\end{array}\right]<0,} \\
& \operatorname{trace}(Z)<1
\end{aligned}
$$

where $Y=K X$.

iii) $\exists X>0, Y, Z>0$ and $V$ such that

$$
\begin{aligned}
& {\left[\begin{array}{ccc}
-\left(V+V^{T}\right) & \star & \star \\
A V+B_{2} Y+X+V & -2 X & \star \\
C_{z} V+D_{z} Y & 0 & -\gamma I
\end{array}\right]<0,} \\
& {\left[\begin{array}{cc}
-Z & \star \\
B_{1} & -X
\end{array}\right]<0,} \\
& \operatorname{trace}(Z)<1
\end{aligned}
$$

where $Y=K V$.

Proof: For proof refer to [13].

It is worth noting that as $V+V^{T}>0, V$ is nonsingular and thus if the LMI (6) is feasible, the state feedback would be derived as $F=Y V^{-1}$. It should also be emphasised that the specific LMI characterisation in (6) enables us to utilise different Lyapunov matrices for each of the involved LMI constraints in the problem. This is because in the LMI (6) the product terms between the matrix $A$ and the Lyapunov matrix have disappeared. In such a case, the control gain can be obtained independent of the Lyapunov matrix. This feature has a significant implication in the design of controllers for systems with parametric uncertainties.

Now, the $\mathscr{H}_{2}$ problem by assuming the control law as $u(t)=K x(t)$ for the system (4), whose matrices belong to the polytope (5), can be cast as the following optimisation problem:

$$
\begin{aligned}
& \text { minimise } \gamma \\
& \text { subject to (6), (7) and (8). }
\end{aligned}
$$

Note also that from the item iii) of Lemma 1, for each vertex $l$, we can employ the following inequalities:

$$
\begin{aligned}
& {\left[\begin{array}{ccc}
-\left(V_{l}+V_{l}^{T}\right) & \star & \star \\
A V_{l}+B_{2} Y_{l}+X_{l}+V_{l} & -2 X_{l} & \star \\
C_{z} V_{l}+D_{z} Y_{l} & 0 & -\gamma I
\end{array}\right]<0,} \\
& {\left[\begin{array}{cc}
-Z_{l} & \star \\
B_{1} & -X_{l}
\end{array}\right]<0,} \\
& \operatorname{trace}\left(Z_{l}\right)<1
\end{aligned}
$$

where $X_{l}>0, Z_{l}>0, Y_{l}$ and $V_{l}$ are variables. However, as seen, solving the optimisation problem in (9) subject to the above inequalities (for $l=1, \cdots, \mathscr{N}$ ) by exploiting different $Y_{l}$ and $V_{l}$ cannot give a unique state feedback $K$. We will use these inequalities in the next subsection to design a sparse row/column-wise SOF for parametric uncertain networked systems by introducing two specific matrix variable transformations. 


\section{Sparse row/column-wise $\mathscr{H}_{2} \mathrm{SOF}$}

Based on the discussions given previously, we specify the requirements of Problem 2 in the following problem.

Problem 3: Given a networked system with the state space representation in Equation (4) involving the parametric uncertainties, design a sparse row/column-wise SOF such that it ensures the $\mathscr{H}_{2}$ performances, i.e. $\left\|T_{w z}\right\|_{2}^{2}<\gamma$, while $\mathrm{S}\left(K_{y}\right) \subseteq \Gamma$, where $\Gamma$ is a priori specified sparse row/column wise structure matrix and $K_{y}$ is the SOF.

The SOF problem can be considered as a constrained state feedback problem; i.e. a state feedback (say $K$ ) which satisfies the additional constraint $K=K_{y} C$ [14]. Effective schemes to address a similar non-convex optimisation problem for the design of an $\mathscr{H}_{\infty}$ SOF and mixed $\mathscr{H}_{2} / \mathscr{H}_{\infty}$ SOF are proposed in [12], [14]. In this paper, unlike [12], [14], the output matrix $C$ belongs to the polytope (5). We now introduce specific LMI decision variables transformations as

$$
\begin{aligned}
& V_{l}=\Sigma_{l} V_{\Sigma} \Sigma_{l}^{T}+\Xi_{l} V_{\Xi} \Xi_{l}^{T}, \\
& Y_{l}=Y_{\Xi} \Xi_{l}^{T},
\end{aligned}
$$

where $V_{\Sigma} \in \mathbb{R}^{(n-p) \times(n-p)}$ and $V_{\Xi} \in \mathbb{R}^{p \times p}$ are symmetric matrices, and $Y_{\Xi} \in \mathbb{R}^{m \times p}$. Besides, $\Sigma_{l}=\operatorname{null}\left(C_{l}\right) \in \mathbb{R}^{n \times(n-p)}$ and $\Xi_{l} \in \mathbb{R}^{n \times p}$ is any matrix that satisfies $C_{l} \Xi_{l}=I$. In general form, $\Xi_{l}$ can be considered as $\Xi_{l}=C_{l}^{\dagger}+\Sigma_{l} \Phi_{l}$, where $\Phi_{l} \in$ $\mathbb{R}^{(n-p) \times p}$ is a given matrix and $C_{l}^{\dagger}=C_{l}^{T}\left(C_{l} C_{l}^{T}\right)^{-1}$. As seen, the matrix transformation proposed in (13) is essentially different from the ones proposed in [12], [14]. Now by letting the variables $V_{l}$ and $Y_{l}$ be (13), the SOF gain can be obtained through the following lemma.

Lemma 2: Let $V_{l}=\Sigma_{l} V_{\Sigma} \Sigma_{l}^{T}+\Xi_{l} V_{\Xi} \Xi_{l}^{T}$ and $Y_{l}=Y_{\Xi} \Xi_{l}^{T}$, then $V_{l}$ is invertible if and only if $V_{\Xi}$ is invertible. Also, in such a case, $Y_{l} V_{l}^{-1}=K_{y} C_{l}$ with $K_{y}=Y_{\Xi} V_{\Xi}^{-1}$.

Proof: Left-multiply $V_{l}=\Sigma_{l} V_{\Sigma} \Sigma_{l}^{T}+\Xi_{l} V_{\Xi} \Xi_{l}^{T}$ by $C_{l}$ to find that $C_{l} V_{l}=V_{\Xi} \Xi_{l}^{T}$. As it is guaranteed that $V_{l}$ is invertible, $C_{l} V_{l}$ is of rank $p$, and thereby $V_{\Xi}$ should have rank $p$. Then, as $V_{\Xi}$ is a full rank square matrix, it is invertible as well. As a result, $\Xi_{l}^{T}=V_{\Xi}^{-1} C_{l} V_{l}$, and thus from (13), we have $Y_{l}=Y_{\Xi} V_{\Xi}^{-1} C_{l} V_{l}$. Now, it can be seen that

$$
Y_{l} V_{l}^{-1}=Y_{\Xi} V_{\Xi}^{-1} C_{l} \triangleq K_{y} C_{l} .
$$

Now the sparse row/column-wise $\mathscr{H}_{2}$ SOF problem, by exploiting LMI approach, can be set as the following optimisation problem:

$$
\begin{aligned}
\text { minimise } & \gamma \\
\text { subject to } & (10),(11),(12), \text { for } l=1, \cdots, \mathscr{N}, \\
& \mathrm{S}\left(Y_{\Xi}\right) \subseteq \Gamma, \mathrm{S}\left(V_{\Xi}\right)=I, \text { and (13). }
\end{aligned}
$$

Remark 1: Matrix $\Phi_{l}$ plays an important role in the proposed method for SOF design. A trivial choice is $\Phi_{l}=0$. However, other choices for $\Phi_{l}$ can be considered, such as $\Phi_{l}=\left(\Sigma_{l}^{T} \Sigma_{l}\right)^{-1} \Sigma_{l}^{T} V_{l} C_{l}^{T}\left(C_{l} V_{l} C_{l}^{T}\right)^{-1}$. However, this choice requires solving the optimisation problem in (9) subject to LMIs in (10)-(12) in advance to find $V_{l}$ associated with each vertex $l$. Clearly, if no solution can be attained by solving
(9), the SOF design problem would not be feasible and no further action is required to be taken for the output feedback problem.

\section{IDENTIFYING FAVOURABLE SPARSE Row/COLUmN-Wise STRUCTURES}

The previous section developed a framework for the design of an $\mathscr{H}_{2}$-based SOF while constraining the structure of the feedback gain. In this section, we aim to address the objective mentioned in Problem 1; i.e. seeking for an optimal subset of available publishers/subscribers in the networked system while the $\mathscr{H}_{2}$-norm degradation of the closed-loop system is minimised relative to the fully distributed topology, or equivalently, finding favourable sparse block row/columnwise SOF gains. This problem can also be seen as searching for the redundant publishers and subscribers of the networked system. We indeed aim to construct a multi-objective optimisation program, where the block row/column sparsity of the SOF gain is directly incorporated into the index function. This is encapsulated in the following problem.

Problem 4: Given a system with the state space representation in Equation (4), find $K_{y}=Y_{\Xi} V_{\Xi}^{-1}$ and $\gamma>0$ in the following optimisation program:

$$
\text { minimise } \quad \gamma+\left\|\Psi_{s} Y_{\Xi}\right\|_{\text {off-row-0 }}+\left\|Y_{\Xi} \Psi_{p}\right\|_{\text {off-col-0 }},
$$

subject to the constraints in (14),

where $Y_{\Xi}$ is a full decision matrix; i.e. $\mathrm{S}\left(Y_{\Xi}\right) \triangleq \Gamma=\mathbf{1}_{m \times p}$, the off-row-O (off-col-O) is a quasi-norm that counts the number of non-zero off-diagonal block rows (columns) of $Y_{\Xi}$, and $\Psi_{s}=\operatorname{diag}\left[\psi_{s, i} I_{m_{i}}\right]_{i=1}^{h}\left(\Psi_{p}=\operatorname{diag}\left[\psi_{p, j} I_{p_{j}}\right]_{j=1}^{h}\right)$, with $\psi_{s, i} \geq 0$ $\left(\psi_{p, j} \geq 0\right)$, is a weighting matrix that implies the emphasis on the off-diagonal block row-sparsity (column-sparsity) of $Y_{\Xi}$, and thus the SOF $K_{y}$. For example, a larger $\psi_{s, i}\left(\psi_{p, j}\right)$ will lead to not employing $i$-th subscriber ( $j$-th publisher) in the control system.

Obviously, an intractable combinatorial search is required to address the optimisation problem above, hence the computation time would grow faster than polynomial, as the order of the networked system system grows [11]. A number of convex approximation of quasi-zero-norms are proposed yet, such as $\ell_{1}$-norm or weighted $\ell_{1}$-norm [15]. In addition, the paper [15] proposes the reweighted $\ell_{1}$ (REL1) minimisation method which is nothing but an iterative program that solves a sequence of weighted minimisation problems, in which at each iteration the weights are updated based on the previous iteration's solution. The REL1 algorithm has recently been used by a number of researchers (e.g., see [16], [8]) for the design of sparse controllers for the distributed systems. Nevertheless, the developed REL1 schemes in these references do not promote row/column-sparsity of the feedback gain which is required in (15). Here, we need to develop a novel method in which the variable selection should amount to the selection of the important groups of variables (block rows and/or columns), rather than important individual variables (elements in the feedback gain).

Remark 2: In the existing literature, a scalar is used to weight the sparsity of the feedback gain; cf. [7], in an 
extended objective function, with the value of this scalar determining the emphasise on the sparsity of the feedback gain. However, in real cases, there may be prior information available about the control network. For example, some communication links can be infeasible or unattractive due to the high implementation costs. In this case, to assist the optimisation-based program proposed for the sparity pattern recognition, it would be advisable to incorporate this a priori knowledge into the optimisation problem by using different scalars for weighting different off-diagonal block rows (columns). This can be implemented simply by specification of diagonal matrices $\left(\Psi_{s}\right.$ and $\left.\Psi_{p}\right)$ of separate weights corresponding to individual off-diagonal block rows (columns).

Let us now recast the objective function of the optimisation problem (15) as follows

Problem 5: Given a system with the state space representation in Equation (1), find $K_{y}=Y_{\Xi} V_{\Xi}^{-1}$ and $\gamma>0$ in the following optimisation program:

$$
\text { minimise } \gamma+f_{s}\left(\Psi_{s} Y_{\Xi}\right)+f_{p}\left(Y_{\Xi} \Psi_{p}\right)
$$

subject to the constraints in (14),

excluding the structural constraint on $Y_{\Xi}$. Here, $f_{s}(\cdot)\left(f_{p}(\cdot)\right)$ denotes the relaxed off-diagonal block-row-sparsity (blockcolumn-sparsity) promoting function.

The following subsection proposes candidates for $f_{s}(\cdot)$ and $f_{p}(\cdot)$.

\section{A. REL1 for row sparsity promoting penalty function}

A convex alternative for the non-convex off-row-0 quasinorm, can be the following function

$$
f_{s}\left(\Psi_{S} Y_{\Xi}\right)=\sum_{i, j, i \neq j}^{h} W_{s, i} \psi_{s, i}\left\|Y_{\Xi, i j}\right\|_{F},
$$

where $\|\cdot\|_{F}$ denotes the Frobenius norm, and similarly

$$
f_{p}\left(Y_{\Xi} \Psi_{p}\right)=\sum_{i, j, i \neq j}\left\|Y_{\Xi, i j}\right\|_{F} \psi_{p, j} W_{p, j},
$$

is a convex approximation for the non-convex off-col-0 quasinorm in (16). Moreover, the update rule for $W_{s, i}$ can be considered as

$$
W_{s, i}^{k}=\frac{1}{\sum_{\substack{j \\ j \neq i}}\left\|K_{y, i j}^{(k-1)}\right\|_{F}+\varepsilon}
$$

where $k$ denotes the current iteration and we use $0<\varepsilon \ll 1$ to provide stability and to ensure that a zero valued off-diagonal block row in $Y_{\Xi}$ does not strictly prevent a non-zero value at the next step. The weighting matrix will be formed as $W_{s}=\left[w_{s, i j}\right]_{h \times h}$, where

$$
w_{s, i j}=\left\{\begin{array}{lc}
\mathbf{0}_{m_{i} \times p_{i}} & \text { if } i=j \\
W_{s, i} \mathbf{1}_{m_{i} \times p_{j}} & \text { otherwise. }
\end{array}\right.
$$

As seen, the weights are updated without considering the Frobenius norm of the block diagonal entries of the SOF gain, because local measurements should not raise the communication cost. It is also worth noting that the obtained relaxed sparsity promoting function do not promote sparsity within the blocks, but at the level of block rows. Similarly, $W_{p, j}$ can be updated as

$$
W_{p, j}^{k}=\frac{1}{\sum_{\substack{i \\ i \neq j}}\left\|K_{y, i j}^{(k-1)}\right\|_{F}+\varepsilon}
$$

Additionally, we form the weighting matrix as $W_{p}=$ $\left[w_{p, i j}\right]_{h \times h}$, where

$$
w_{p, i j}= \begin{cases}\mathbf{0}_{m_{i} \times p_{i}} & \text { if } i=j \\ W_{p, j} \mathbf{1}_{m_{i} \times p_{j}} & \text { otherwise. }\end{cases}
$$

Remark 3: It is worth mentioning that minimising the number of subscribers and publishers or either of them have the possibility of promoting a completely decentralised control structure which is equal to a control network with no subscriber and publisher.

\section{B. An algorithm for solving Problem 5}

Now, define the matrix $\Pi=\left[\pi_{i j}\right]_{h \times h}$ with

$$
\pi_{i j}= \begin{cases}\mathbf{0}_{m_{i} \times p_{i}} & \text { if } i=j \\ \mathbf{1}_{m_{i} \times p_{j}} & \text { otherwise. }\end{cases}
$$

The optimisation problem in (16), by letting $f_{S}\left(Y_{\Xi}\right)$ and $f_{p}\left(Y_{\Xi}\right)$ as (17) and (18), respectively, is equivalent to

$$
\text { minimise } \gamma+\operatorname{trace}\left(\Pi^{T} \Psi_{s} \mathscr{Y}_{1}\right)+\operatorname{trace}\left(\mathscr{Y}_{2} \Psi_{p} \Pi^{T}\right)
$$

subject to the constraints in (15),

$$
\begin{aligned}
& -\mathscr{Y}_{1} \leq W_{s} \circ Y_{\Xi} \leq \mathscr{Y}_{1}, \\
& -\mathscr{Y}_{2} \leq W_{p} \circ Y_{\Xi} \leq \mathscr{Y}_{2},
\end{aligned}
$$

where $\circ$ denotes the Hadamard product (entry-wise product). For addressing the above convex problem and identifying a sparse row/column-wise SOF, the following algorithm is proposed.

\section{Algorithm 1:}

1) Given $\varepsilon>0, \alpha>0, \Psi_{s}>0$ and $\Psi_{p}>0$, initialise $W_{s}=$ $\left[w_{s, i j}\right]_{h \times h}, W_{p}=\left[w_{p, i j}\right]_{h \times h}$, with $w_{s, i j}$ and $w_{p, i j}$ as in (20) and (22), respectively, by letting $W_{s, i}=1$ and $W_{p, j}=1$, $k=1$ and $K_{y}^{k}=0$.

2) Solve the minimisation problem (23) to obtain $K_{y}^{\star}=$ $Y_{\Xi}^{\star} V_{\Xi}^{\star}{ }^{-1}$.

3) Update $W_{s, i}^{k}$ and $W_{p, j}^{k}$ using the update rules in (19) and (21), respectively, and form $W_{s}=\left[w_{s, i j}\right]_{h \times h}$ and $W_{p}=$ $\left[w_{p, i j}\right]_{h \times h}$ as in (20) and (22), respectively.

5) If $\left\|K_{y}^{\star}-K_{y}^{k}\right\| \leq \alpha$ go to Step 6 , else $K_{y}^{k}=K_{y}^{\star}, k=k+1$ and return to Step 2.

6) Let the unnecessary block rows and columns of $K_{y}^{\star}$ be zero and return $\Gamma^{\star}=\mathrm{S}\left(K_{y}^{\star}\right)$.

Eventually, in order to find the $\mathscr{H}_{2}$ structured SOF with the identified $\Gamma^{\star}$, we turn to the minimisation problem in (14). 

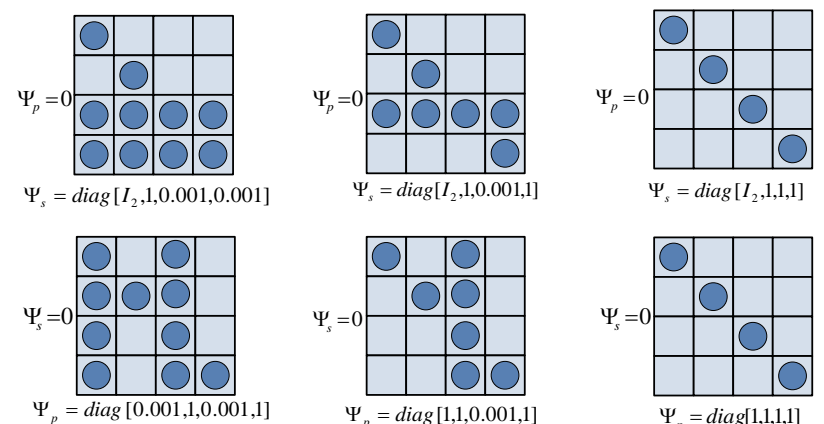

$\Psi_{p}=\operatorname{diag}[1,1,1,1]$

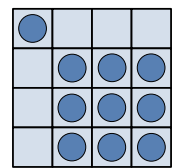

$\Psi_{s}=\operatorname{diag}\left[I_{2}, 0.001,0.001,0.001\right]$ $\Psi_{p}=\operatorname{diag}[1,0.001,0.001,0.001]$
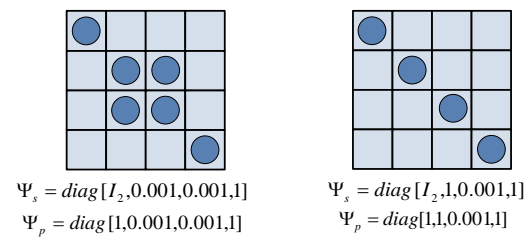

Fig. 2. Structure of block row/column-sparse SOF gains for different values of $\Psi_{s}$ and $\Psi_{p}$

\section{NUMERICAL EXAMPLES}

Consider a decentralised interconnected system, presented in [4], that consists of four subsystems:

$$
A=\left[\begin{array}{cc|c|cc|c}
-1 & 2 & 1 & -1 & 0 & 1 \\
0 & 1 & 2 & 0 & 0 & 0 \\
\hline 0.5 & -0.5 & -2 & 0 & 1 & 1 \\
\hline 1 & -1 & -1 & -2 & 0.4 & 0 \\
0 & 0 & 0 & 1 & -3 & 1 \\
\hline 2 & 0 & -1 & -2 & 0 & -4
\end{array}\right]
$$$$
B_{2}=\left[\begin{array}{cc|c|c|c}
1 & 0 & 0 & 0 & 0 \\
0 & 0.5 & 0 & 0 & 0 \\
\hline 0 & 0 & 1 & 0 & 0 \\
\hline 0 & 0 & 0 & 2 & 0 \\
0 & 0 & 0 & 1 & 0 \\
\hline 0 & 0 & 0 & 0 & 2
\end{array}\right], C=\left[\begin{array}{ll|l|ll|l}
0 & 1 & 0 & 0 & 0 & 0 \\
\hline 0 & 0 & 1 & 0 & 0 & 0 \\
\hline 0 & 0 & 0 & 1 & 0 & 0 \\
\hline 0 & 0 & 0 & 0 & 0 & 1
\end{array}\right],
$$$$
B_{1}=I_{6}, C_{z}=\left[\begin{array}{c}
I_{6} \\
0_{5 \times 6}
\end{array}\right], D_{z}=\left[\begin{array}{c}
0_{6 \times 5} \\
I_{5}
\end{array}\right] \text {. }
$$

We additionally assume that there exists an uncertainty in the entry $(4,6)$ of the output matrix $C$ by up to $\pm 50 \%$ of its nominal value. As seen this interconnected system is fully coupled and open-loop unstable. Solving the convex problem in (14), by letting $\Gamma=\mathbf{1}_{m \times p}$ and assuming a block diagonal structure for $V_{l}, l=1,2$ as $V_{l}=\Sigma_{l} V_{\Sigma} \Sigma_{l}^{T}+\Xi_{l} V_{\Xi} \Xi_{l}^{T}$ where $V_{\Sigma} \in \mathbb{R}^{2 \times 2}$ and $V_{\Xi}=\operatorname{diag}\left[V_{\Xi, i}\right]_{i=1}^{4} \in \mathbb{R}^{4 \times 4}$, with $V_{\Xi, i} \in \mathbb{R}$, are symmetric matrices, and $\Phi_{l}=0$, results in a true $\mathscr{H}_{2}$-norm of 3.7689 (with nominal $C$ ). We now exploit Algorithm 1 with $\alpha=0.01$ and $\varepsilon=0.001$. By increasing $\Psi_{s}$ and $\Psi_{p}$ from zero, the number of block rows and columns with non-zero off-diagonal blocks in the SOF gain decreases; see Fig. 2. Once the sparsity structures of controllers are identified for different $\Psi_{s}$ and $\Psi_{p}$, the resulting patterns are used to solve (23), by identified $\Gamma$, in order to obtain the $\mathscr{H}_{2}$ block row/column-sparse structured controllers.

\section{CONCLUSIONS}

This paper has been devoted to the design of optimal sparse block row/column-wise feedback gains for dynamical systems. Firstly, we developed an explicit LMI-based method for the design of $\mathscr{H}_{2} \mathrm{SOF}$ gain for systems with polytopic uncertainty, which is capable of incorporating additional structural constraints on the feedback gain matrix. Following this, an iterative process for the identification of favourable sparse block row/column-wise SOF gains has been proposed. Then, in order to find the optimal structured feedback gain, we solved the developed $\mathscr{H}_{2}$-based SOF problem subject to the identified pattern. This scheme has an immediate application in publisher/subscriber networked systems for selecting a subset of available publishers and/or subscribers, aimed at, for example, reducing the communication costs etc. In addition, we have not involved the Lyapunov function used for checking system performances in the controller variables, resulting in less conservatism compared to the socalled quadratic approach. This technique can also widen the applicability region of the proposed static output feedback design method for parametric uncertain systems. However, it is worth noting that increasing the number of matrix variables in the optimisation-based controller design problem may increase the computation time. Nevertheless, it is evident that, it is practically not necessary to wait for the termination criterion in the REL1 algorithms, and mostly they can identify the favourable patterns after a few iterations. A numerical example has clearly demonstrated the effectiveness of the proposed approach.

\section{REFERENCES}

[1] A. Zecevic and D. Siljak, "Global low-rank enhancement of decentralized control for large-scale systems," IEEE Transactions on Automatic Control, vol. 50, no. 5, pp. 740-744, 2005.

[2] D. Siljak, Decentralized control of complex systems. Dover Publications, 2012.

[3] X. Wang and M. Lemmon, "Event-triggering in distributed networked control systems," IEEE Transactions on Automatic Control, vol. 56 , no. 3, pp. 586-601, 2011.

[4] M. Staroswiecki and A. M. Amani, "Fault-tolerant control of distributed systems by information pattern reconfiguration," International Journal of Adaptive Control and Signal Processing, 2014.

[5] M. Razeghi-Jahromi and A. Seyedi, "Stabilization of networked control systems with sparse observer-nontroller networks," IEEE Trans. Autom. Control, to be published.

[6] R. Arastoo, Y. GhaedSharaf, M. V. Kothare, and N. Motee, "Optimal state feedback controllers with strict row sparsity constraints," in American Control Conference (ACC), 2016. IEEE, 2016, pp. 19481953

[7] F. Lin, M. Fardad, and M. Jovanovic, "Augmented lagrangian approach to design of structured optimal state feedback gains," IEEE Trans. Autom. Control, vol. 56, no. 12, pp. 2923-2929, 2011.

[8] D. M. Zoltowski, N. Dhingra, F. Lin, and M. R. Jovanovic, "Sparsitypromoting optimal control of spatially-invariant systems," in American Control Conference (ACC), 2014. IEEE, 2014, pp. 1255-1260.

[9] M. Fardad, F. Lin, and M. R. Jovanovic, "Sparsity-promoting optimal control for a class of distributed systems," in Proc. the American Control Conference, San Francisco, CA, USA, 2011, pp. 2050-2055.

[10] M. Van De Wal and B. De Jager, "A review of methods for input/output selection," Automatica, vol. 37, no. 4, pp. 487-510, 2001.

[11] S. Schuler, U. Münz, and F. Allgöwer, "Decentralized state feedback control for interconnected systems with application to power systems," Journal of Process Control, vol. 24, no. 2, pp. 379-388, 2014.

[12] A. Argha, S. W. Su, A. Savkin, and B. G. Celler, "Mixed $H_{2} / H_{\infty}$ based actuator selection for uncertain polytopic systems with regional pole placement," International Journal of Control, no. just-accepted, pp. 1-23, 2017.

[13] A. Argha, S. W. Su, and A. Savkin, "Optimal actuator/sensor selection through dynamic output feedback," in Decision and Control (CDC), 2016 IEEE 55th Conference on. IEEE, 2016, pp. 3624-3629. 
[14] J. Rubió-Massegú, J. M. Rossell, H. R. Karimi, and F. PalaciosQuiñonero, "Static output-feedback control under information structure constraints," Automatica, vol. 49, no. 1, pp. 313-316, 2013.

[15] E. J. Candes, M. B. Wakin, and S. P. Boyd, "Enhancing sparsity by reweighted $\ell_{1}$ minimization," Journal of Fourier analysis and applications, vol. 14, no. 5-6, pp. 877-905, 2008.

[16] M. Fardad and M. R. Jovanovic, "On the design of optimal structured and sparse feedback gains via sequential convex programming," in American Control Conference (ACC), 2014. IEEE, 2014, pp. 24262431. 